
\title{
Analysis of the Effect of Variation of Reference Channel on Neuronal Activity for Motor Imagery Electroencephalography Signal
}

\author{
Ashutosh Gupta', Sujata Pandey' and J. L. Raheja² \\ 'Department of Electronics and Communication Engineering, Amity University, Noida, Uttar Pradesh -201313, \\ India; agupta5@amity.edu \\ ${ }^{2}$ CSIR-Central Electronics Engineering Research Institute, Pilani, Rajasthan - 333031, India; \\ jagdishraheja@rediffmail.com
}

\begin{abstract}
Objectives: Topographic map has been studied to observe the amount of neuronal activity at various electrode locations. Methods/Statistical Analysis: Electroencephalography (EEG) refers to recording of electrical activities (potential or current density) produced by neurons. A raw EEG signal obtained through electrodes placed in a specific pattern on the scalp. One of the electrodes needs to be selected as a Reference, relative to which electrical activity at all other electrodes shall be measured. It is usually said that electrodes at "inactive" regions of the scalp should be selected as the reference but this paper deals with analyzing what happens if any other electrode is selected as reference as there is no single best reference for all the purposes. The magnitude of electrical activity at various electrodes is indicated by color codes so that it is visually distinguishable. Findings: The difference in topography of EEG rhythms shall be observed and the extent up to which this is correct with different electrodes as reference would be seen. We observe that asymmetry arises if electrodes are at right or left rather than at Center line. We also observe that the electrodes which are at similar potential as that of the reference is indicated by Red color. Application/Improvements: The electrodes at very high potential than the reference are indicated by yellow whereas blue indicates medium activity w.r.t. the reference. These signals may be used for BCI application.
\end{abstract}

Keywords: Electroencephalography ( EEG), Neurons, Open Vibe Signal Processing

\section{Introduction}

\subsection{Obtaining EEG}

Neurons use variation in current density or potential across the membranes in order to establish communication with one another. This electrical activity can be recorded by placing electrodes in a particular fashion on the scalp. A reference electrode needs to be selected from which the potential of any specific electrode can be subtracted in order to determine if a particular electrode is at a higher, lower or equal potential as the reference. Although the Spatial Resolution of EEG is poor but the temporal resolution of EEG signal is excellent $(<1 \mathrm{~ms})$ which makes it the most commonly used Non-Invasive technique for Brain Imaging 1 . SNR of the EEG signal is improved using temporal filters ${ }^{2}$. There is no specific best reference electrode as there are some shortcomings associated with each.

The main contribution of this paper is to analyze the magnitude of neuronal activity at various electrode locations when various electrodes are taken as the reference.

${ }^{*}$ Author for correspondence 
There has been no indicative study suggesting a particular reference electrode to be perfect as there are certain limitations associated with each and for different applications, different electrodes may be used as the reference.

The paper is organized as inputting a raw EEG signal which has a very low value of SNR and pre-processing it by Temporal Filters. Now this filtered signal is sent for further processing. The difference in electrical activity of neurons due to selection of different electrodes as reference is observed. The regions where electrical activity reduces or increases shall be seen with respect to the reference.

\subsection{Selection of Reference Electrode}

If either right or left earlobe is selected as the reference, although the topography of EEG rhythms is very close to the true value, the electrodes closer to the reference side experience a decrease in amplitude of the EEG signal which is undesirable ${ }^{3}$.

If both the right and left earlobe electrodes are linked and used as the reference,

- asymmetry is created

- output EEG waveform turns out to be distorted

- current starts flowing within the wires linking the earlobes

- the intracranial currents get affected

- EEG potentials that get recorded is inaccurate.

- Low amplitude EEG is observed in temporal region.

\section{Methodology}

The process involved in re-referencing the electrode and further analyzing it are included in this section. The flowchart includes various steps involved in carrying out the procedure is shown in figure 1. Firstly a raw EEG signal is taken. A motor imagery signal has been taken from datasets available online. This signal contains noise and we need to de-noise it in order to carry out the analysis in a proper manner. This is done with the Help of Temporal Filters which enhance the SNR of signal. As per our con- venience, we can select any electrode as a Reference. We have taken various electrodes as reference and tried to analyze the effect of the same. Topographic maps are used to study the activities at various regions of the brain.

\subsection{Flowchart}

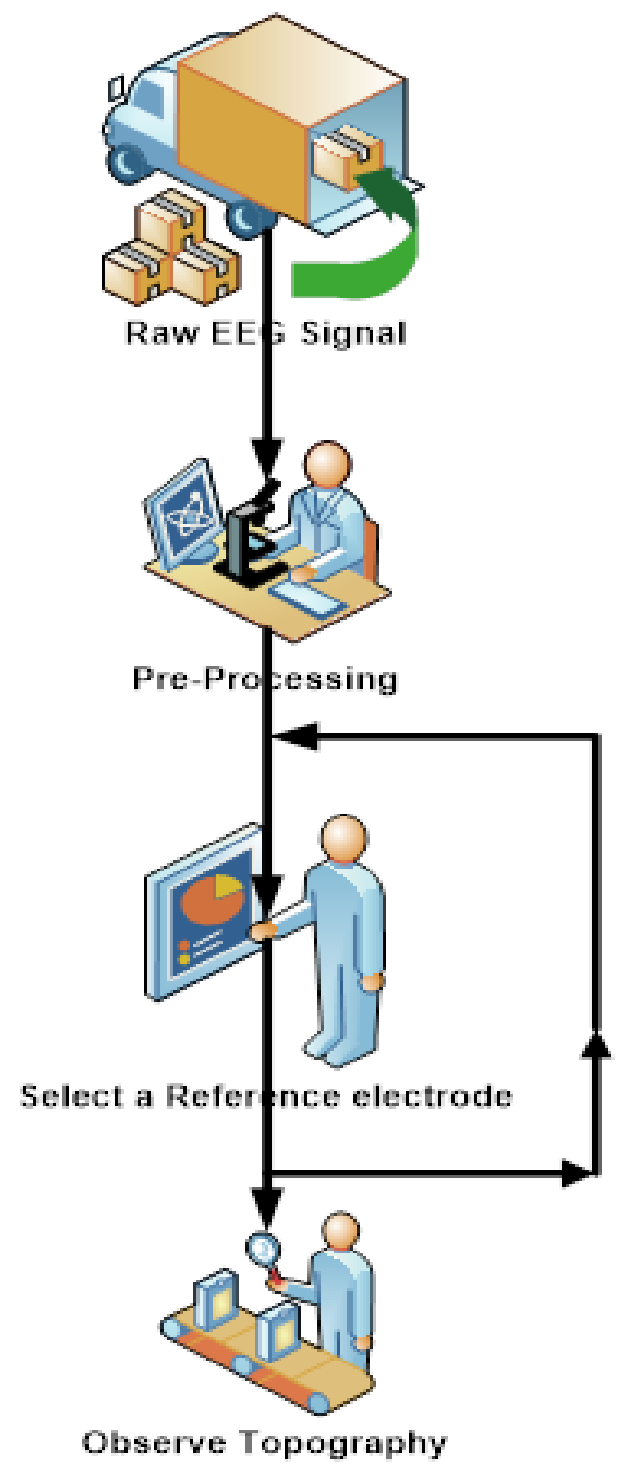

Figure 1. Process involved in Re-Referencing of scalp electrodes. 


\subsection{Procedure}

1. Take a raw EEG signal as shown in figure 2. We have taken a .gdf Motor Imagery Dataset obtained online.

2. This dataset contains recordings from electrodes: $\mathrm{Cz}, \mathrm{C} 3, \mathrm{C} 4, \mathrm{~F} 3, \mathrm{~F} 4, \mathrm{P} 3, \mathrm{P} 4, \mathrm{O} 1, \mathrm{O} 2$

3. Pre-process this signal using temporal filter shown in figure 3, to improve its SNR and thus de-noise it using OpenViBE 4

4. Send this pre-processed signal for further processing. Power spectral density and time frequency map can be observed as shown in figure 4 and figure 5 respectively.
5. Take one of the electrodes as the reference

6. Obtain the topographic maps for each reference electrode.

7. Observe the topography obtained through spline interpolation algorithm ${ }^{\frac{5}{5}}$ of the potentials at various locations

8. Analyse the measured activity at various electrode locations

\section{Analysis}

A topographic map is used to visually distinguish the potential difference between reference and electrode under consideration ${ }^{6}$. These maps make use of color cod-

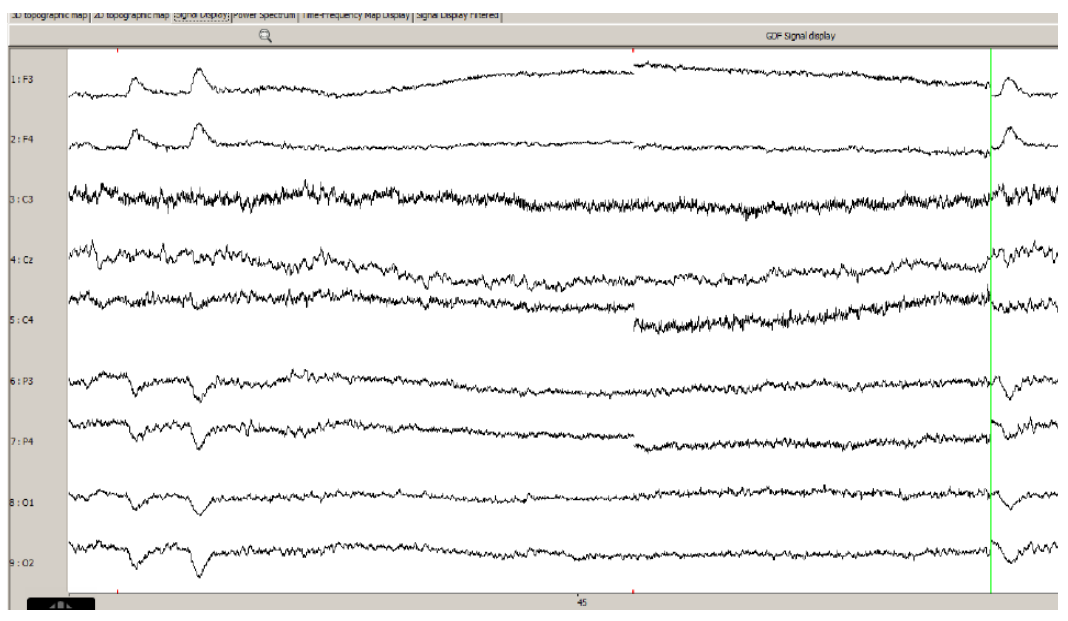

Figure 2. Raw EEG Signal (Source: openvibe.inria.fr ${ }^{4}$ ).

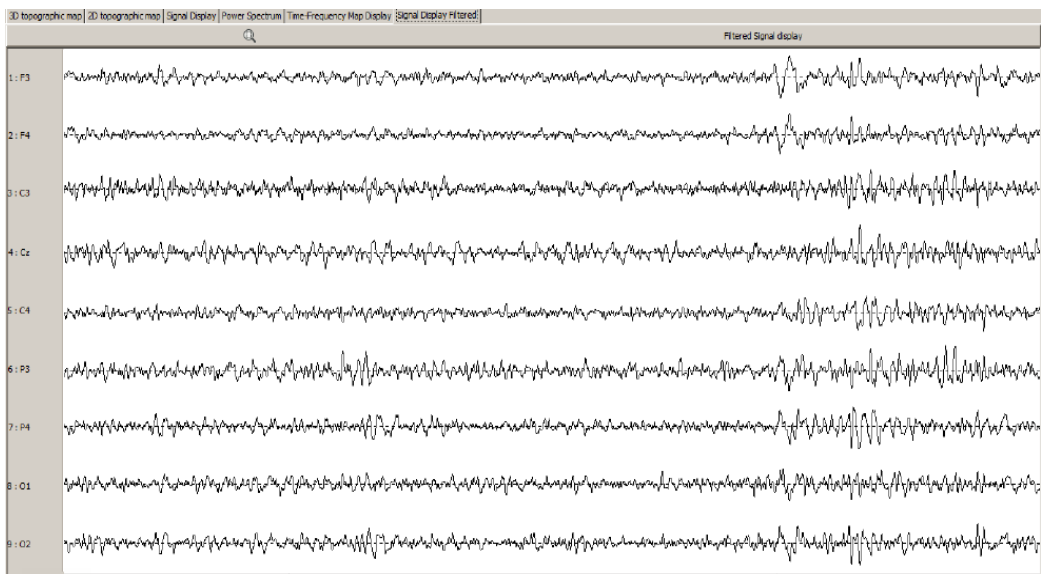

Figure 3. Filtered Signal. 


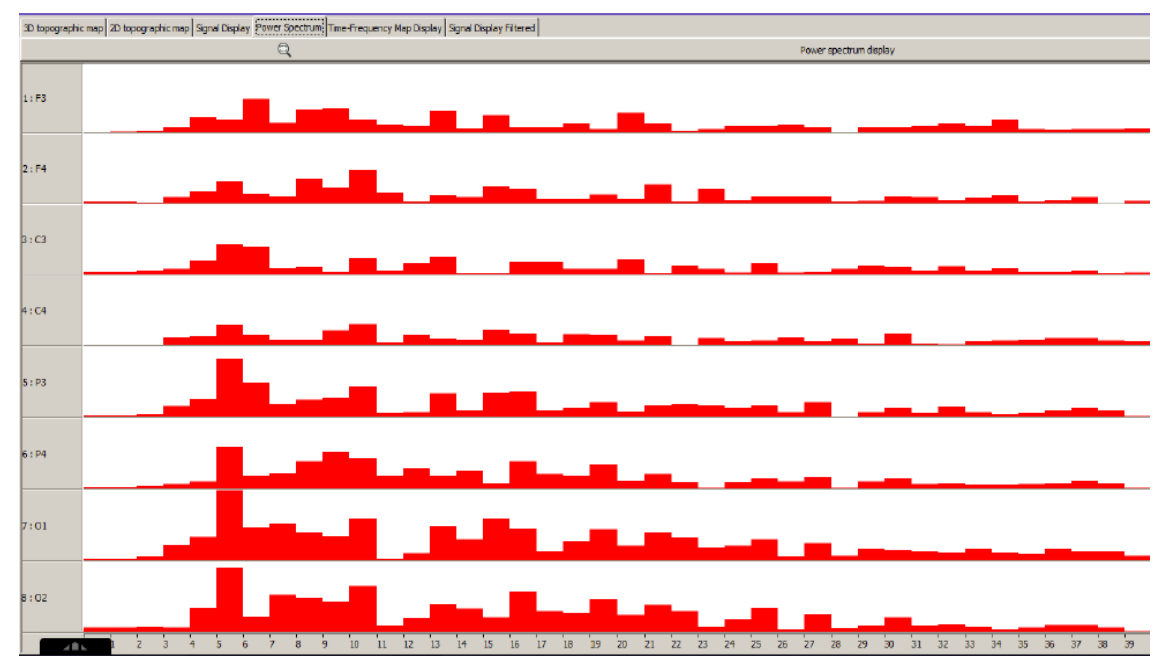

Figure 4. Power Spectral Density.

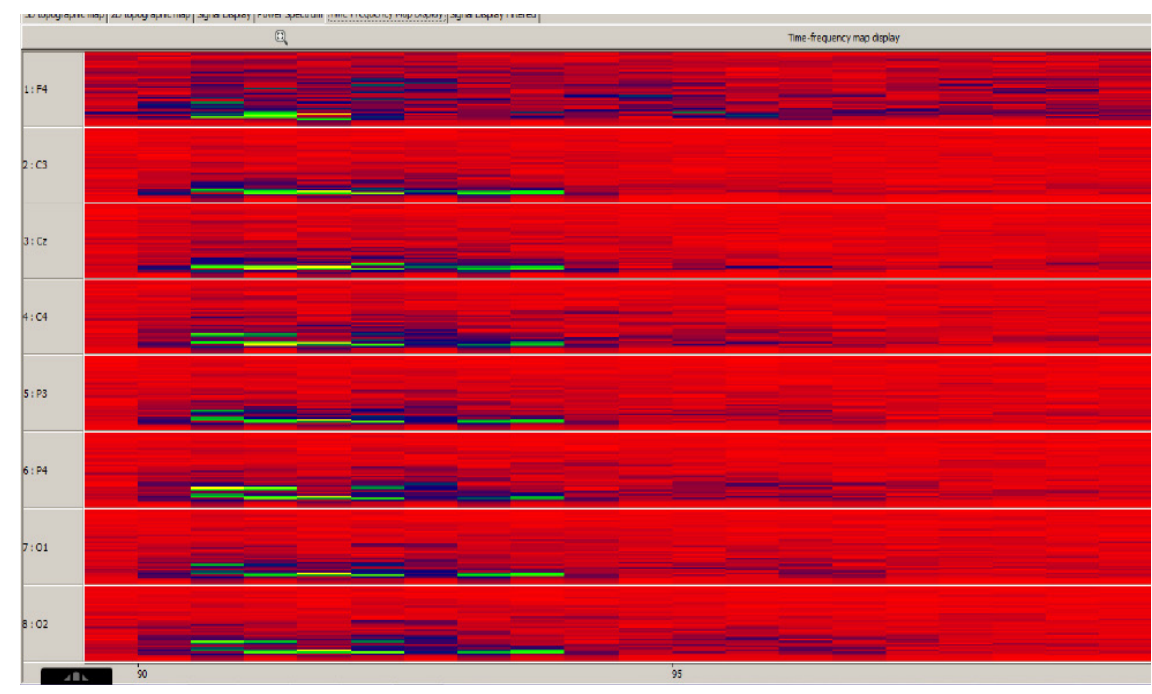

Figure 5. Time Frequency Map.

ing. Yellow and its shades indicate higher electrical activity, blue indicate medium activity and red indicate lower electrical activity. Figure 6 (a) shows the Topographic map of Cortical electrode $\mathrm{Cz}$ and Channel locations and 6 (b) shows the Topographic maps of Cortical electrodes C3 and $\mathrm{C} 4$ located at left and right respectively. Topographic maps of Frontal electrodes F3 and F4 has been shown in figure 7, and Topographic maps of Occipital electrodes $\mathrm{O} 1$ and $\mathrm{O} 2$ shown in figure 8. And figure 9 shows the Topographic maps of Parietal electrodes P3 and P4.

The magnitude of neuronal activity in various regions keeping different electrodes as a reference has been 

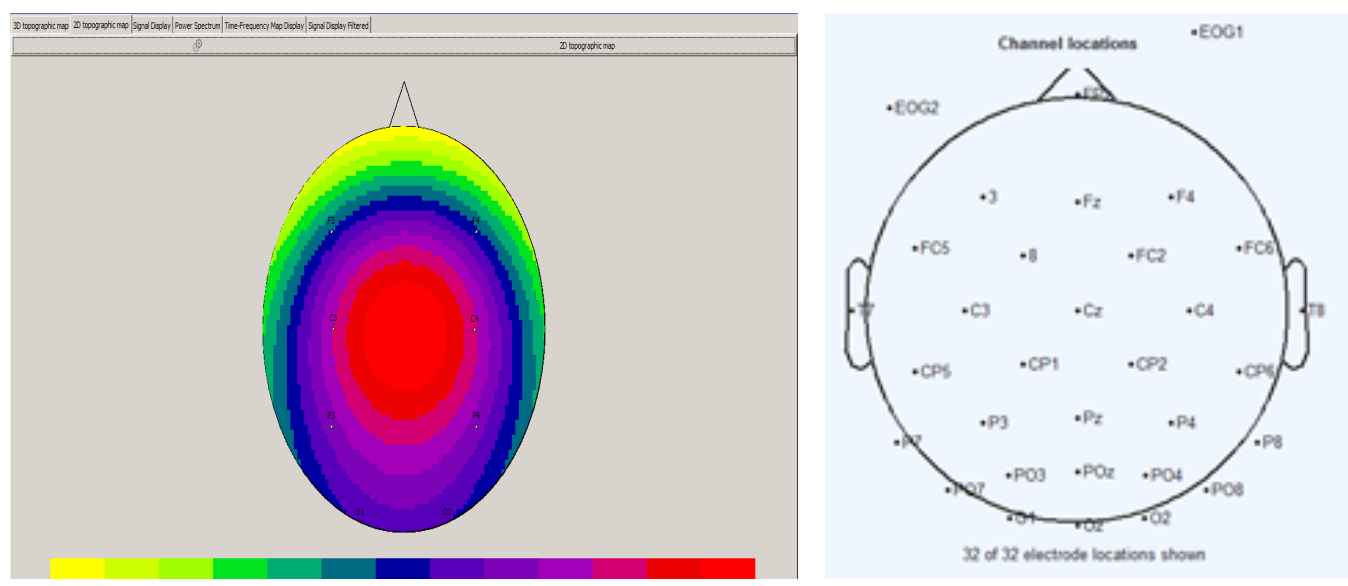

(a)

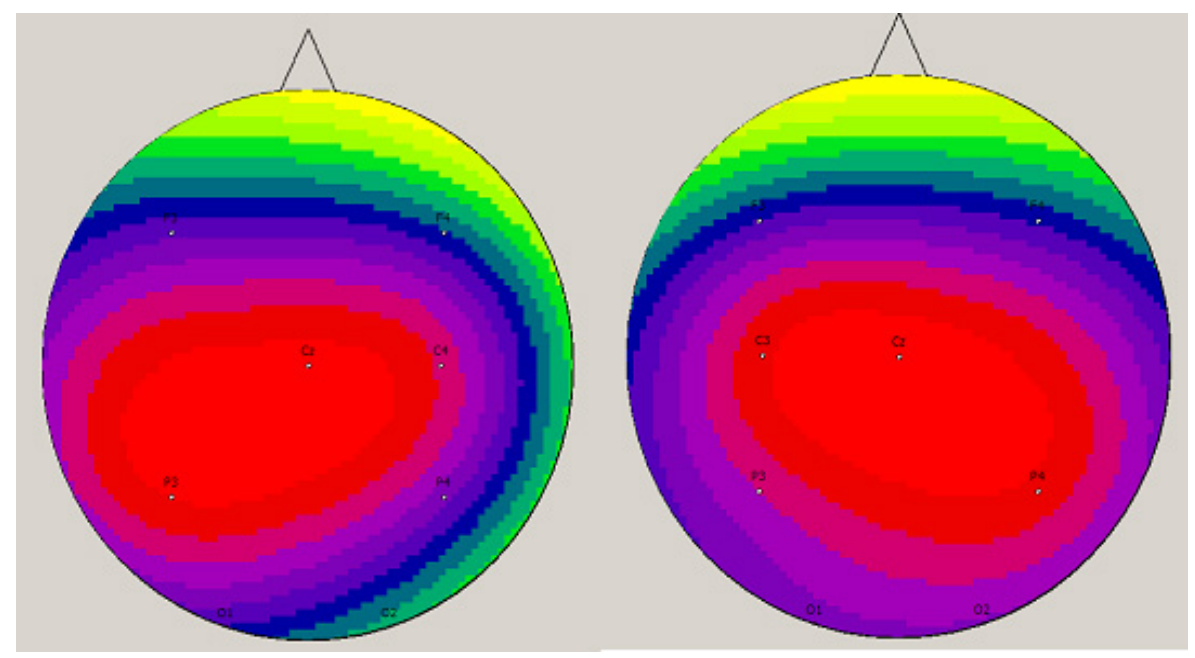

(b)

Figure 6. (a)Topographic map of Cortical electrode $\mathrm{Cz}$ and Channel locations. (b) Topographic maps of Cortical electrodes C3 and C4 located at left and right respectively.

observed using the topographic maps and the results are formulated in Table 1. We observe that the electrodes which are at similar potential as that of the reference are indicated by Red color. The electrodes at very high potential than the reference are indicated by yellow whereas blue indicates medium activity.
Figure 2 displays the raw Motor Imagery EEG signal taken online from Datasets available.

Figure 3 displays the filtered signal obtained by passing the raw EEG signal through Temporal Filter. We have used a Butterworth Band Pass Filter with frequency range 


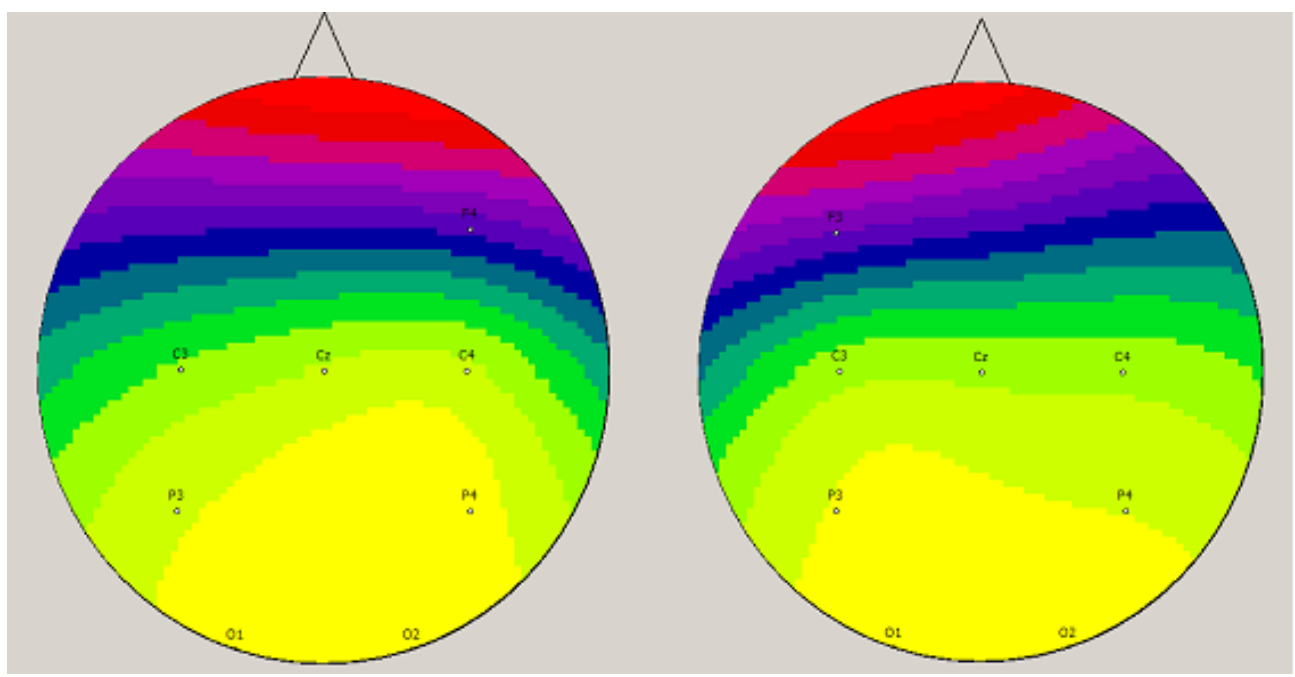

Figure 7. Topographic maps of Frontal electrodes F3 and F4.

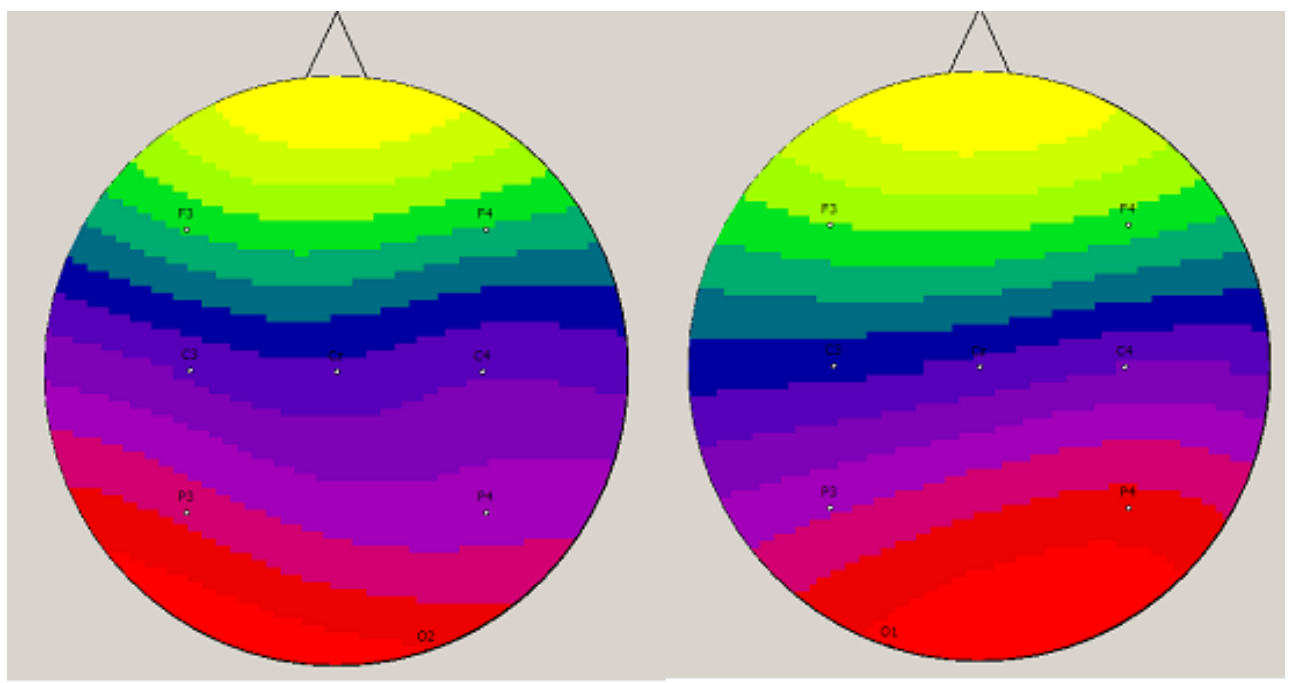

Figure 8. Topographic maps of Occipital electrodes $\mathrm{O} 1$ and $\mathrm{O} 2$.

from 4 to $40 \mathrm{~Hz}$. This is done to improve the SNR of raw signal

Figure 4 is the Power Spectral density of EEG signal used for analysis.

Figure 5 shows the Time - Frequency map which is obtained by plotting Power spectral Density versus time. As an EEG signal is non-stationary, its frequency com- ponent varies w.r.t time and thus a time-frequency map gives its best representation.

Figure 6(a) and Figure 6(b) indicate the neuronal activities at various electrode locations when Cortical Electrodes $\mathrm{Cz}, \mathrm{C} 3$ and $\mathrm{C} 4$ are selected as the reference. Also, exact channel locations are shown in Fig. 6(a) We observe that when $\mathrm{Cz}$ electrode, located at center of the 

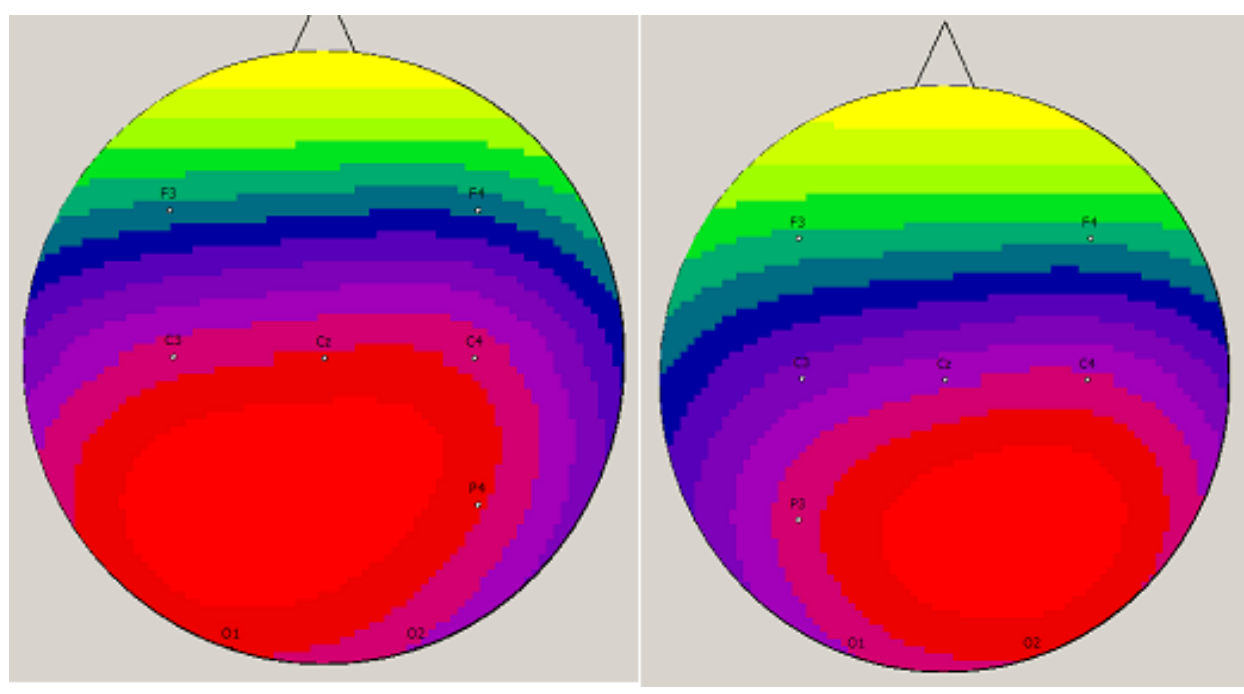

Figure 9. Topographic maps of Parietal electrodes P3 and P4.

scalp is chosen as the reference, no asymmetry is created whereas if we select C3 located at left or C4 located at right the color coding clearly shows asymmetry while interpolating potentials at various electrodes.

When frontal electrodesF3 and F4 are selected as reference as indicated in Figure - 7, all other electrodes except frontal electrodes show high amount of neuronal activity w.r.t the reference.

Figure 8 clearly indicates that when occipital electrodes $\mathrm{O} 1$ and $\mathrm{O} 2$ are selected as reference, frontal electrodes show high activity and Parietal electrodes show low activity whereas cortical electrodes show medium activity from which we can infer that cortical electrodes are at similar potential as the occipital electrodes.

Figure 9 shows neuronal activities when Parietal electrodes are taken as reference. Very high neuronal activity is not shown by any electrode in this case.

Thus, a topographic map very clearly indicates the neuronal activities in a very simple to understand manner. Such maps were obtained by Splice Interpolation method.

Table 1. Electrical activities at various electrodes

\begin{tabular}{|c|c|c|c|}
\hline $\begin{array}{c}\text { Reference } \\
\text { electrode }\end{array}$ & $\begin{array}{c}\text { High activity } \\
\text { electrodes }\end{array}$ & $\begin{array}{c}\text { Medium activity } \\
\text { electrodes }\end{array}$ & $\begin{array}{c}\text { Low activity } \\
\text { electrodes }\end{array}$ \\
\hline $\mathrm{Cz}$ & $\mathrm{F} 3, \mathrm{~F} 4$ & $\mathrm{O} 1, \mathrm{O} 2, \mathrm{P} 3, \mathrm{P} 4$ & $\mathrm{C} 3, \mathrm{C} 4$ \\
\hline $\mathrm{C} 3$ & $\mathrm{~F} 3, \mathrm{~F} 4$ & $\mathrm{O} 1, \mathrm{O} 2, \mathrm{P} 3, \mathrm{P} 4$ & $\mathrm{Cz}, \mathrm{C} 4$ \\
\hline $\mathrm{C} 4$ & $\mathrm{~F} 3, \mathrm{~F} 4$ & $\mathrm{O} 1, \mathrm{O} 2, \mathrm{P} 3, \mathrm{P} 4$ & $\mathrm{Cz}, \mathrm{C} 3$ \\
\hline $\mathrm{F} 3$ & $\begin{array}{c}\mathrm{O} 1, \mathrm{O} 2, \mathrm{P} 3, \mathrm{P} 4 \\
\mathrm{C} 3, \mathrm{C} 4, \mathrm{Cz}\end{array}$ & $\mathrm{F} 4$ & - \\
\hline
\end{tabular}


Table 1 Continued

\begin{tabular}{|c|c|c|c|}
\hline $\mathrm{F} 4$ & $\begin{array}{r}\mathrm{O} 1, \mathrm{O} 2, \mathrm{P} 3, \mathrm{P} 4, \\
\mathrm{C} 3, \mathrm{C} 4, \mathrm{Cz}\end{array}$ & $\mathrm{F} 3$ & - \\
\hline $\mathrm{O} 1$ & $\mathrm{~F} 3, \mathrm{~F} 4$ & $\mathrm{Cz}, \mathrm{C} 3, \mathrm{C} 4$ & $\mathrm{O} 2, \mathrm{P} 3, \mathrm{P} 4$ \\
\hline $\mathrm{O} 2$ & $\mathrm{~F} 3, \mathrm{~F} 4$ & $\mathrm{Cz}, \mathrm{C} 3, \mathrm{C} 4$ & $\mathrm{O} 1, \mathrm{P} 3, \mathrm{P} 4$ \\
\hline $\mathrm{P} 3$ & - & $\mathrm{F} 3, \mathrm{~F} 4$ & $\begin{array}{l}\mathrm{O} 1, \mathrm{O} 2, \mathrm{P} 4, \\
\mathrm{Cz}, \mathrm{C} 3, \mathrm{C} 4\end{array}$ \\
\hline $\mathrm{P} 4$ & - & $\mathrm{F} 3, \mathrm{~F} 4$ & $\begin{array}{l}\mathrm{O} 1, \mathrm{O} 2, \mathrm{P} 4, \\
\mathrm{Cz}, \mathrm{C} 3, \mathrm{C} 4\end{array}$ \\
\hline
\end{tabular}

\section{Conclusion}

Topographic map has been studied to observe the amount of neuronal activity at various electrode locations. If the difference between potential at reference and at a particular electrode comes out to be positive, the shades of yellow and green indicate it. Further as we approach blue color, the potential difference of electrode w.r.t reference is negligible. Then, as the difference turns out to be negative, the color code changes to violet and then red at extreme end. Further, we observe that a cortical electrode either at left, right or center gives similar color coding in topographic maps. Similarly, Frontal (F3, F4), Occipital (O1, O2) and Parietal (P3,P4) irrespective of their right or left location give similar color coding in topographic maps. Also we observe that the color orientation is a bit more towards the side at which reference electrode is located, i.e. asymmetry is created when the electrode is located at left or right side of the scalp. In applications where the activity of a particular side has to be focused upon, such kind of reference can be used

\section{References}

1. Wallace E, Ruedade L, Reilly RB, Doherty CP. The limitations of neuroimaging - a possible role for quanti- tative Electroencephalography. Proceedings of the Irish Neurological Association, Cork. 61. 2012.

2. Antara Bhattacharya, Bawane NG, Nirkhi SM . Brain computer interface using EEG signals. IEEE research paper. p. 2 2 A.S.011.

3. Lehmann D. Gevins A. Rémond (Eds): Principles of spatial analysis. In: Handbook of Electroencephalography and Clinical Neurophysiology. Volume 1: M Michel CM, Seeck M, Landis T. Spatio-temporal dynamics of human cognition. News Physiol Sci, 1999;14:206-214. chel CM, Seeck, M, Murray MM. The sp and Magnetic Signals. Amsterdam: Elsevier, 1987; 309-54

4. Yann Renard, Fabien Lotte, Guillaume Gibert, Marco Congedo, Emmanuel Maby, Vincent Delannoy, Olivier Bertrand, Anatole Lécuyer. OpenViBE: An Open-Source Software Platform to Design, Test, and Use Brain-Computer Interfaces in Real and Virtual Environments".2010 February; 19( 1) :35-53

5. Law SK, Nunez PL, Wijesinghe RS. High-resolution EEG using spline generated surface laplacians on spherical and ellipsoidal surfaces. IEEE Transactions on Biomedical Engineering. 1993; 40(2):145-53

6. Hisaki Ozaki, Satoru Watanabe, Hiroya Suzuki, Topographic EEG changes due to hypobaric hypoxia at simulated high altitude. Electroencephalography and Clinical Neurophysiology. 1995 May; 94(5):349-56. 